mers, cellulose, hemicelluloses and the peptidoglycan of the bacterial cell wall. They refer, moreover, to observations of an interaction between galactomannan and a bacterial polysaccharide. These relationships, they suggest, must play a part in determining the organization and the mechanical properties of cell walls and other structures.

\section{PROTEIN SYNTHESIS}

\section{Interchangeable Factors}

from our Molecular Genetics Correspondent

THE universal nature of the genetic code and the ability of the protein synthetic apparatus of one type of organism to translate the messenger RNAs of another species in vitro point to the development of the code as a very early stage in evolution. The sole differences between the dictionaries of code words for amino-acids in different organisms seem to be confined to the punctuation signals; the AUG codon which initiates protein synthesis responds to a formylated methionyl-tRNA in bacteria but to a methionyl-tRNA with a free amino-group in the cells of higher organisms. The termination signals are probably the same in all organisms, although there is some evidence which suggests that the UGA codon may be used for the amino-acid cysteine in eukaryotes.

This common evolution is further emphasized in an article from Zasloff and Ochoa (Proc. US Nat. Acad. Sci., 69, 1796 ; 1972) which shows that the protein factors which initiate protein synthesis are interchangeable between a variety of organisms. In Escherichia coli cells, there are three such factors; f3 which seems to direct $30 \mathrm{~S}$ subunits of the ribosome to bind to initiation sites on messenger RNA, f2 which assists the binding of fmet-tRNA $A_{f}$ to this complex, and f1 which seems to play some part in both processes. These factors can be washed off $30 \mathrm{~S}$ subunits with ammonium chloride and counterparts have been found in some eukaryotic cells; in other higher organisms, the proteins needed for initiation are found in the supernatant and do not seem to be associated with the ribosome subunits.

Zasloff and Ochoa have previously isolated a protein factor from the brine shrimp, Artemia salina, which seems to correspond to factor f 2 of $E$. coli, the only obvious difference in its action being that it does not require GTP for its activity. The factor has a molecular weight of about 100,000 - which is much larger than its bacterial analogue--and shares with $\mathrm{f} 2$ a sensitivity to the reagent $\mathrm{N}$-ethyl-maleimide which reacts with free sulphydryl groups. Zasloff and Ochoa have now prepared a mixed system which contains the ribosomes of the shrimp, but in which they substitute the initiation factors of mouse L cells, rat liver, or rabbit reticulocytes in place of the Artemia factor. They find that these heterologous systems work perfectly well; and so does the reverse exchange system in which the Artemia factor promotes binding of the initiator tRNA to the ribosomes of rat liver. The eukaryotic factors cannot be replaced by bacterial factor $\mathrm{f} 2$, nor can they replace $\mathrm{f} 2$ with bacterial ribosomes. But the interchangeability of the factors from these different eukaryotes indicates that not only have the signals which start proteins been conserved in evolution, but also the mechanisms which respond to them.

Another heterologous initiation complex has been formed by Noll, Noll and Lingrel (ibid., 1843) ; they have assayed the ability of the ribosomes, initiator tRNA, and initiation factors of $E$. coli to form complexes with mammalian globin messenger RNA. These complexes are readily formed, and the bound formyl-methionine can be released by puromycin, which indicates that it enters its usual binding site on the ribosome. The first amino-acidafter the initiator methionine which is later removed-in both globin chains is valine; if the ribosomes are binding to the correct initiation sites, therefore, valyl-tRNA should be bound to the complex. But although binding takes place with a mixture of aminoacyltRNAs and amino-acids can be incorporated into protein, valyl-tRNA does not bind specifically.

This implies that the ribosomes have formed an initiation complex at the wrong site on the messenger. But binding seems to be specific and has the same characteristics as binding to the correct initiation sites on messengers used in $E$. coli. This suggests that the sequence on mRNA to which ribosomes bind may be longer than the AUG codon itself and is different in $E$. coli and mammals; the sequence to which the $E$. coli ribosomes bind on globin mRNA must fortuitously resemble the natural initiation sites in $E$. coli messengers.

This is important for two reasons. First, it confirms the idea that AUG codons within a message may be distinguished from those which act as initiators by their context; the nature of this context may be different in prokaryotes and eukaryotes, perhaps because in the first mRNA is translated even as it transcribed from DNA, whereas in the cells of higher organisms the completed messenger must be transported from nucleus to cytoplasm.

Even more interesting is the possibility that the binding site on the globin mRNA can be sequenced by the now tried technique of degrading the mRNA in the complex with ribonuclease; the sequence bound to the messenger is protected and can be recovered and sequenced. The chief problem in using this technique is simply lack of sufficient messenger; so far, only the binding sites of RNA phages have been determined and these have not thrown much light on the initiation process, perhaps because these molecules are not true cellular RNAs and represent a special case. Globin mRNA can be prepared in large amounts and it will be intriguing to see whether there really is only one sequence on mammalian mRNA to which ribosomes bind or whether there is less specificity. And one important caution which these results point to is that specific binding in

\title{
Neuraminidase Stimulates Cell Division
}

A VARIETY of factors-including serum, proteases, insulin and, of course, tumour viruses-can induce confluent monolayers of cultivated cells, which have ceased to divide, to begin further rounds of cell division. And because proteases immobilized on beads are as effective as enzyme in solution it seems likely that at least some of these stimulatory factors exert their effects by causing changes in the chemistry and/or architecture of the cell surface. In next Wednesday's Nature New Biology (August 16), Vaheri, Ruoslahti and Nordling add another enzyme, neuraminidase, to the list of molecules capable of triggering multiplication in confluent cultures of cells. That neuraminidase has this effect is not surprising because sialic acid is a component of cell surface glycolipids and glycoproteins which are attacked by this enzyme.
Vaheri and his colleagues found that neuraminidase from either $V$. cholerae or $\mathrm{Cl}$. perfringens induces DNA synthesis and mitosis in confluent cultures of chick fibroblasts to about 30 per cent at most of the extent obtained with optimal amounts of insulin. Stimulation of cell division by neuraminidase was proportional to the concentration of the enzyme over the range $0.02 \mu \mathrm{g} /$ $\mathrm{ml}$. to about $0.5 \mu \mathrm{g} / \mathrm{ml}$., and at these concentrations no protease activity could be detected contaminating the neuraminidase. Vaheri et al. also measured the uptake of 2-deoxy-D- ${ }^{3} \mathrm{H}$ glucose and report that after exposure to neuraminidase it is enhanced by some two to three-fold. They suggest that all these data indicate "that sialic acid containing membrane molecules are critical for growth control and for the transmission of the stimulatory effect of neuraminidase, insulin and trypsin". 